\title{
Move more for life: the protocol for a randomised efficacy trial of a tailored-print physical activity intervention for post-treatment breast cancer survivors
}

Camille E Short $^{1 *}$, Erica L James ${ }^{1}$, Afaf Girgis ${ }^{2}$, Patrick Mcelduff ${ }^{3}$ and Ronald C Plotnikoff ${ }^{4}$

\begin{abstract}
Background: Due to early detection and advances in treatment, the number of women surviving breast cancer is increasing. Whilst there are many positive aspects of improved survival, breast cancer survival is associated with many long-term health and psychosocial sequelae. Engaging in regular physical activity post-diagnosis can reduce this burden. Despite this evidence, the majority of breast cancer survivors do not engage in regular physical activity. The challenge is to provide breast cancer survivors with appealing and effective physical activity support in a sustainable and cost-effective way. This article describes the protocol for the Move More for Life Study, which aims to assess the relative efficacy of two promising theory-based, print interventions designed to promote regular physical activity amongst breast cancer survivors.
\end{abstract}

Method and design: Breast cancer survivors were recruited from across Australia. Participants will be randomised into one of three groups: (1) A tailored-print intervention group, (2) a targeted-print intervention group, or (3) a standard recommendation control group. Participants in the tailored-print intervention group will receive 3 tailored newsletters in the mail over a three month period. Participants in the targeted-print group will receive a previously developed physical activity guidebook designed specifically for breast cancer survivors immediately after baseline. Participants in the standard recommendation control will receive a brochure detailing the physical activity guidelines for Australian adults. All participants will be assessed at baseline, and at 4 and 10 months post-baseline. Intervention efficacy for changing the primary outcomes (mins/wk aerobic physical activity; sessions/exercises per week resistance physical activity) and secondary outcomes (steps per day, health-related quality life, compliance with physical activity guidelines, fatigue) will be assessed. Mediation and moderation analyses will also be conducted.

Discussion: Given the growing number of cancer survivors, distance-based behaviour change programs addressing physical activity have the potential to make a significant public health impact.

Trial registration: Australian New Zealand Clinical Trials Registry (ANZCTR) identifier: ACTRN12611001061921

\footnotetext{
*Correspondence: Camille.Short@Newcasltle.edu.au

${ }^{1}$ School of Medicine and Public Health, Priority Research Centre for Health

Behaviour, Priority Research Centre for Physical Activity and Nutrition,

University of Newcastle, Callaghan, Australia

Full list of author information is available at the end of the article
}

\section{Biomed Central}

(c) 2012 Short et al.; licensee BioMed Central Ltd. This is an Open Access article distributed under the terms of the Creative Commons Attribution License (http://creativecommons.org/licenses/by/2.0), which permits unrestricted use, distribution, and reproduction in any medium, provided the original work is properly cited. 


\section{Background}

Due to earlier detection and advances in treatment, more and more women are surviving breast cancer each year [1]. Whilst improved survival is duly welcomed, breast cancer survivors are faced with both short and long-term health and psychosocial sequelae [2], including fatigue, reductions in physical and cognitive functioning, reductions in bone health, lymphedema, weight gain and mood disturbances [3-6]. Compared to the general (non-cancer) population, breast cancer survivors are at an increased risk of comorbid chronic conditions and death from both cancer and non-cancer causes [7]. As such, there is a growing need for effective cancer recovery services that can help to improve the quality of life of breast cancer survivors and negate the associated health burdens and risks [8].

One promising cancer recovery strategy is the promotion of regular physical activity (PA) [9,10]. Evidence from health outcome trials suggests that regular PA can address both the psychological and physiological burdens presented after breast cancer diagnosis and treatment $[11,12]$. Furthermore, observational research suggests that regular PA may also have an impact on survival, with breast cancer survivors who are active after treatment having a lower risk of cancer recurrence, co-morbidities and death from all causes compared to those who are less active, regardless of cancer stage [13-15]. In recognition of these benefits, detailed exercise prescription guidelines for cancer survivors have been published by professional bodies in both Australia and North America $[9,10,16]$.

There is also new evidence that addressing the pattern of activity is important, with unique metabolic consequences associated with prolonged sedentary behaviour, regardless of total activity time [15,17]. Despite this evidence, the majority of breast cancer survivors are not sufficiently active for health $[17,18]$ and efforts to encourage regular PA and reductions in sitting time are not a routine part of the cancer treatment or rehabilitation process [19-22].

Whilst over 70 PA intervention studies have been conducted with cancer survivors, the majority have been atheoretical face-to-face programs conducted during the treatment phase [23-26]. Whilst these interventions have been efficacious in improving important outcomes for cancer survivors, there is a need for more sustainable, less resource intensive approaches that can support survivors beyond the initial treatment phase [27-29]. Such programs should be grounded in behaviour change theory, and address the unique determinants of PA adoption and maintenance in the post-treatment breast cancer population [30,31].

The purpose of this study is to evaluate the relative efficacy of two promising distance-based approaches (targeted and tailored print interventions) for promoting PA among post-treatment breast cancer survivors compared to a standard recommendation control group. In targeted-print interventions, irrelevant information is reduced by providing individuals with materials targeted to a particular subgroup they belong to (e.g., breast cancer survivors) [32]. In tailored-print interventions, computer technology is utilised to provide individuals with personalised advice based on information specific to them (derived from individual assessment) [32]. Both approaches have been put forth as low-cost, evidencebased alternatives to resource intensive face-to-face programs [32,33], but little information exists about the relative efficacy and the cost/benefit of these approaches in the physical activity domain.

Some theories of information processing, such as The Elaboration Likelihood Model [34], suggest that people are more likely to process information in a way that is conducive to behaviour change, if it is personally relevant to them. Based on this model, we hypothesise that individuals randomised into either the targeted or tailored print groups will experience significantly greater improvements at each timepoint on all primary and secondary outcomes compared to the standard recommendation control group. Furthermore, given the greater level of personalisation of materials in the tailored-print condition, we expect participants in the tailored-print group to experience greater improvements across PA outcomes compared to participants in the targeted-print group. This hypothesis relies on the assumption that breast cancer survivors are a somewhat heterogeneous group in terms of determinants (i.e., demographics, socialcognitive and ecological factors) of PA behaviour change.

\section{Methods \\ Design}

This study is a nationally-based, three-arm randomised controlled trial (RCT), testing the relative efficacy of two distance-based PA interventions (tailored and targeted print) compared to a standard recommendation control group. Participants will complete data collection at baseline, 4 months and 10 months. Ethics approval was obtained from the University of Newcastle Human Research Ethics Committee (H-2010-11-3). The RE-AIM framework [35] will serve to guide the dissemination of this program in terms of adoption, implementation and maintenance. The conduct and reporting of this study will adhere to the Consolidating Standards of Reporting Clinical Trials (CONSORT) guidelines [36] and to the Reporting Standards for Studies of Tailored Interventions [37]. The study flow chart is presented in Figure 1 (note: recruitment for this study is complete).

\section{Participants \\ Selection criteria}

Female breast cancer survivors who are over the age of 18 and who have finished "active" cancer treatment (defined as surgery, chemotherapy and/or radiotherapy), who can 


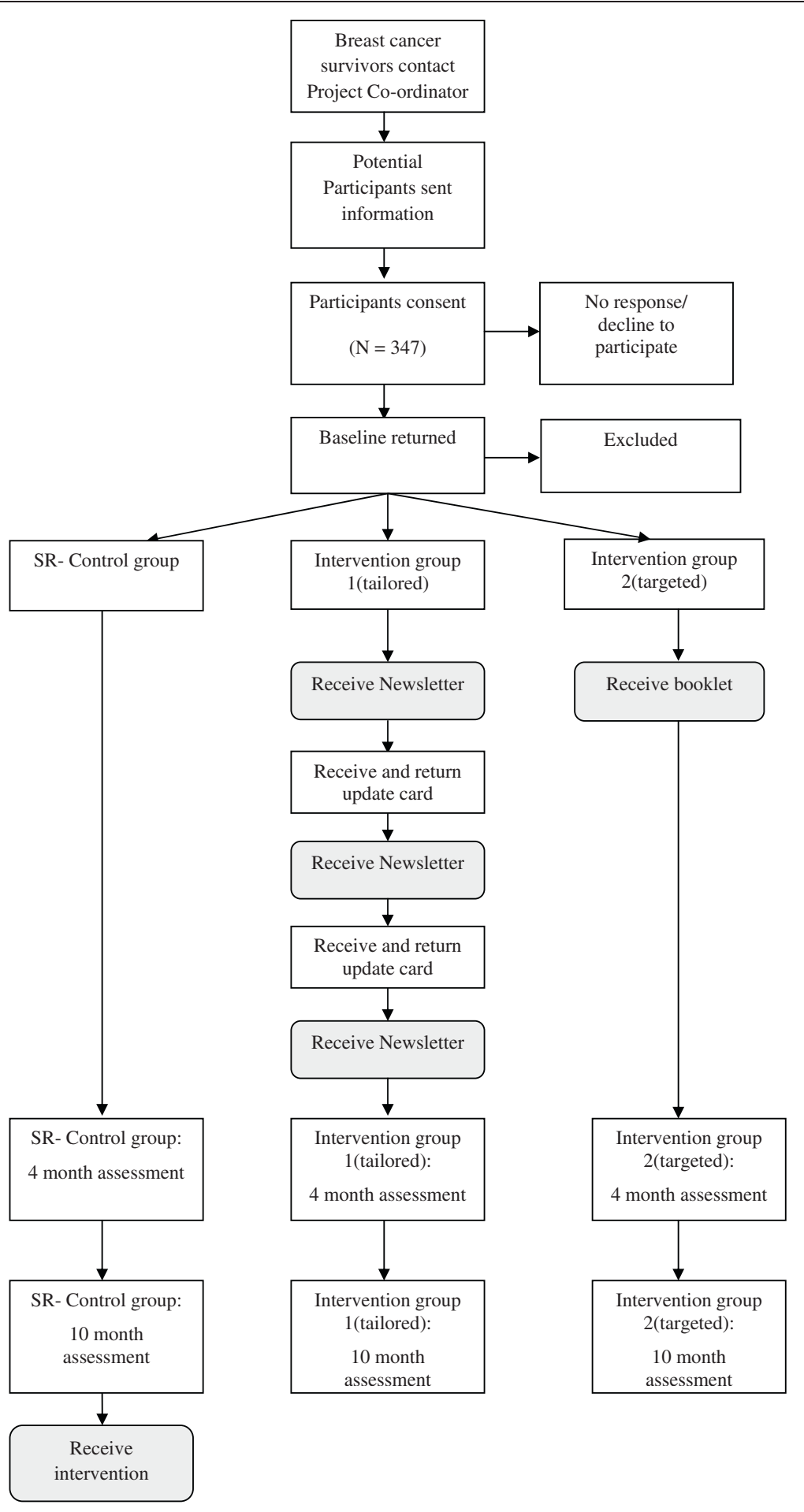

Figure 1 Study flow chart.

read and write in English were eligible to participate in the study. All potential participants were required to complete a physical activity readiness questionnaire (PAR-Q; [38]), to screen participants and identify those requiring review from their doctor prior to study enrolment.

\section{Recruitment}

Participants were recruited using convenience sampling methods from a range of sources across Australia. Specifically, this involved (1) asking organisations (e.g., The Breast Cancer Network Australia, The Cancer Council, 
YWCA Encore) and health professionals (e.g., breast care nurses) with direct contact with breast cancer survivors to disseminate information about the study on behalf of the research team; (2) promoting the study at events potentially relevant to eligible participants (e.g. breast cancer forums); and (3) snowballing recruitment (inviting participants to pass on study information to potentially eligible friends and acquaintances).

\section{Randomisation}

The randomisation sequence will be generated by a statistician (PM) using SAS 9.2 statistical software. An equal number of participants will be randomised to each group (1:1:1) using a randomised block design, with a block size of six, to ensure the study groups are balanced $[39,40]$.

Participants, identified only by their ID number, will be randomised by an administrative staff member into groups upon receipt of their baseline survey. All project team members will be blinded to this process and participant details will remain de-identified until participant allocation is completed. Participant blinding is not possible due to the difference in delivery schedule of the two interventions.

\section{Statistical power and sample size}

The study's primary analysis will be the comparison of selfreported PA behaviour (i.e. mins/week of aerobic exercise and sessions/week of resistance training) between the three groups, from baseline to the 4 month time-point. Assuming a small-moderate correlation $(r=0.4)$ between baseline and post-intervention, to detect a mean difference of 0.5 standard deviation between study groups (smallmedium effect size) for the main dependent outcome (i.e., PA behaviour) at post-intervention [41] the required sample size is 100 participants per group, allowing for a 20\% loss to follow-up (power $=0.80$; alpha $=0.01$ ). An alpha of 0.01 was used to adjust for multiple comparisons.

As a secondary consideration, we also ensured that this sample size would be adequate to detect a clinically significant change in step counts per day (2000 steps per day [42], standard deviation of 3500 [43]) and found that we would be adequately powered to detect meaningful changes in both self-report and objective PA outcomes.

\section{Outcomes}

A pen-and-paper questionnaire is completed at baseline, 4 months post baseline (immediate post- tailored intervention follow-up), and 10 months post-baseline (7 month post tailored-intervention follow-up). At each of these time-points, participants will be asked to wear a pedometer for seven days and complete a step count diary.

\section{Primary outcome}

The primary outcome variables, minutes of PA (aerobic) per week and average number of sessions/exercises per week (resistance) will be assessed using an adapted version [44] of the validated Godin Leisure-Time Exercise Questionnaire (GLTEQ) [45]. The adapted version will incorporate a resistance training (RT) measure $[44,46]$ that asks participants to report the frequency (times per week) and duration (average times per session) of resistance training activities on average over the past month. The original measure has been found to be both reliable and valid [47].

\section{Secondary outcomes}

Step counts Average daily step counts [48] will be estimated based on at least three days of pedometry, which is sufficient to reliably estimate pedometer-determined PA [49,50]. Participants will be instructed to zero the pedometer and record their accumulated steps at the end of each day for seven days, using the step count diary provided. The step count diary will also be used to record instances where the pedometer was intentionally removed (e.g. swimming) or when the participant forget to wear the device. Where reported, step count equivalents for non-ambulatory activities (e.g. swimming, cycling) will be calculated and added to the step count total using the method outlined by Miller et al. [51].

Adherence to PA guidelines PA type, frequency and duration measured by 8 items from the adapted version of the GLTEQ $[44,46]$ will be used to calculate whether or not participants are meeting the PA guidelines for cancer survivors [10].

Sedentary behaviour Sedentary behaviour is measured using a validated five item scale asking about time spent sitting (hours and minutes) each day during the week and on the weekend in the following situations (a) while travelling to and from places; (b) while at work; (c) while watching television; (d) while using a computer from home; and (e) in leisure time not including watching television (e.g. visiting friends, dining out) [52].

Health related quality of life Quality of life is measured using version 4 of the internationally validated 37item FACT-Breast measurement system (FACT-B) [53]. The FACT-B is multidimensional, consisting of subscales measuring cancer specific aspects of physical well-being, emotional well-being, social well-being, functional wellbeing, and 10-items measuring breast cancer specific concerns. 
Fatigue Fatigue is measured using the validated 13-item FACIT (Functional Assessment of Chronic Illness Therapy) Fatigue scale, which assesses self-reported tiredness, weakness and difficulty conducting usual activities [54].

\section{Social cognitive mediators of physical activity}

Hypothesized social cognitive mediators of PA behaviour are assessed using previously published, validated instruments where possible. Some items were adapted to make them more appropriate for use in this study. The adaptations were based on our own qualitative research and formative research in the field (e.g. [55]) and were tested for face validity using a small convenience sample $(n=5)$ of post-treatment breast cancer survivors. In each survey, the time referent used for the items is framed based on the timing of the proceeding follow-up survey (i.e. the baseline survey time referent is "the past/next four months" and the four and ten month follow-up surveys time referent is "the past/next six months").

Outcome expectations Outcome expectations is measured using 5 general items from the validated exercise pros subscale [56] with 6-additional items developed for this study based on formative research among breast cancer survivors [55,57], including our own qualitative research and information provided by experts in the field. The items in the scale assess the extent that individuals agree or disagree $(1=$ strongly disagree to $5=$ strongly agree) that participating in regular PA over the next 4 month would for them: reduce tension or manage stress; increase confidence about one's health; help to sleep better; have a more positive outlook; help control weight; regain lost strength; prevent a cancer recurrence; be enjoyable; increase fatigue; increase joint pain or result in lymphoedema. An example item includes "Over the next four months, participating in regular PA will help me prevent a cancer recurrence."

Outcome expectancies Outcome expectancies will be assessed by asking participants to rate how important each of the outcome expectations are to them (e.g. "For me, reducing joint pain is") on a 3 -point scale ( $1=$ unimportant; 2 important; 3 very important). This scale has been utilised and tested in prior research $[44,58]$.

Self efficacy Task self-efficacy will be assessed using 4items developed [57] and evaluated [59] in previous studies with breast cancer survivors and 3 additional items developed for this study to assess task-self efficacy for resistance training activities. The items assess the participant's level of confidence $(1=$ not at all confident to 5 = extremely confident) that over the next 4 months they can: walk for 20 minutes without stopping; jog for 10 minutes without stopping; climb 3 flights of stairs; exercise for 20 minutes at a level hard enough to cause an increase in heart rate; do 6 wall push ups in a row; do one small session of resistance training including 6 different exercises; and do yoga for 60 minutes (Example item: "Over the next four months, I can do 6 wall push ups in a row").

Barrier self-efficacy will be assessed using 12-items based on previous scales used in chronic disease populations (7 items developed and tested by Rogers' et al. [59] among breast cancer survivors and 5 items developed and tested by Plotnikoff et al. among diabetes patients $[56,60]$ ) and one item ("when I can't notice any improvements in my body") developed for this study based on formative research. Participants will be asked to rate their confidence ( $1=$ not at all confident to $5=$ extremely confident) that they can participate in regular PA over the next four months when: they lack the discipline to exercise; exercise is not a priority; the weather is bad; feeling tired; lack time; do not enjoy exercising; do not have someone to encourage them to exercise; in a bad mood or feeling depressed; have to do it alone; can't notice any improvements in fitness; can't notice any improvements in body; feel stiff and sore; and feel ill.

Behavioural capability Behavioural capability is measured using 6-items assessing specific components of PA knowledge and skill that were developed for this study. Participants will be asked to rate on a 5-point likert scale ( 1 = strongly disagree to $5=$ strongly agree) how much they agree with each of the statements: I know how to warm up and cool down before/after an exercise session; I have a good idea of what type of PA to do to gain health benefits; I have a good idea of how hard I should engage in PA to gain health benefits; I have a good idea of how much PA I should do to gain health benefits; I have the skills I need to engage in aerobic physical activities; and I have the skills I need to engage in resistance-based physical activities.

Environment Social support is assessed using the 15item social support for exercise habits scale [61]. Participants are asked to rate how often during the past four months their friends and family (separately) supported them/discouraged them to exercise in a variety of ways. Response options range from 1 (none) to 5 (very often). An example item is: "During the past four months, my friends gave me encouragement to stick with my exercise program". The perceived built environment will be assessed using an adapted version of the 7-item IPAQ environmental module [62]. Participants will be asked to rate how much they agree or disagree $(1=$ strongly disagree to $5=$ strongly agree) with the following statements: most of the houses in my neighbourhood are detached houses; many shops, stores, markets or other places to buy things I need are within easy walking distance of my home; my 
home is within a 10-15 minute walk to a bus or train station; there are footpaths on most of the streets in my neighbourhood; there are facilities to bicycle in or near my neighbourhood; my neighbourhood has several free or low cost recreation facilities; and the crime rate in my neighbourhood makes it unsafe to go on walks at night.

Self control and performance Self-regulation will be assessed using a 12-item scale developed for use among older adults [63]. The items measure six subscales of self-regulation (self-monitoring, goal setting, eliciting social support, reinforcements, time management, relapse prevention) and can be combined to produce an overall score [63]. An example item is "Over the past 4 months, how often did you rearrange your schedule to ensure you had time for physical activity". Response options range from 1 (never) to 5 (very often).

Action planning will be assessed using 4-items developed by Rise et al. [64] and adapted by Rhodes et al. [65] (to say "physical activity" instead of "exercise"). Participants will be asked to rate the following statements according to their plans over the next two weeks $(1=$ no plans to 5 detailed plans): I have made plans concerning 'when' I am going to engage in regular PA; I have made plans concerning 'where' I am going to engage in regular PA; I have made plans concerning 'what' kind of regular PA I will engage in; I have made plans concerning 'how' I am going to get to a place to engage in regular PA.

\section{Socio-demographics}

The following socio-demographic data will be collected: date of birth, marital status, parental status, living arrangement, country of birth, education, employment, income, internet access, health insurance status and geographical location.

\section{Health status and cancer history}

At baseline and each follow-up time point (where applicable), participants are asked five questions about their health status (physical limitations, perceived weight, menopause status, co-morbidities) and nine questions about their cancer diagnosis (age at diagnosis, cancer stage, treatment type, and prognosis).

\section{Process evaluation}

Participant evaluation of the intervention materials will be measured using 15 multiple choice items and one openended question, included in the immediate post intervention follow-up questionnaire. The 15 multiple choice items were purpose-designed by the research team and are based on the Elaboration Likelihood Model (ELM) [34], which is often utilised to explain the effects of health communication interventions. An example item includes: "how personally relevant was the health information you received" ( $1=$ not at all relevant to $5=$ very relevant). The open ended question provides participants with a chance to make comments about the intervention materials.

\section{Procedure}

Potential participants were asked to contact the project co-ordinator to express their interest in participating in the study. Potential participants were then provided with an information statement and a consent form and asked to return it to the project team within two weeks. Information was resent at two weeks if no response was received.

Participants will be asked to complete a pen-and-paper questionnaire, wear a pedometer for seven days and complete a pen-and-paper step count diary at baseline, four and ten months from baseline (Figure 1). Participants will be instructed to return the pedometer with the written materials using a reply paid envelope as soon as possible after each assessment period is complete. Participants who do not return the baseline questionnaire, and step count diary and pedometer within two weeks will receive one reminder call from the project co-ordinator. Participants who do not return the assessment materials within three weeks after this reminder call will be excluded from the trial.

Upon receipt of the baseline questionnaire, an administrative assistant will allocate participants using the ID number written on the questionnaire into one of three groups using the randomly generated allocation sequence provided by the statistician. Participants will be sent intervention or standard recommendation materials within three weeks of allocation.

Participants in the tailored-print intervention group will be sent additional intervention materials at 6 weeks and 12 weeks post baseline and update cards (3-item update card), 4 weeks and 8 weeks post base-line and asked to return them to the research team using a reply paid envelope within 7 days. Participants in the standard recommendation group will receive one tailored newsletter and a pdf version of the targeted guidebook after completion of the10 month follow-up survey (Figure 1).

\section{Interventions}

Targeted-print intervention Participants randomised into this group will receive a copy of a theory-based exercise guidebook developed specifically for promoting physical activity among breast cancer survivors. This guidebook was developed for use and evaluated in a previous study [66] and has been described in detail elsewhere [67]. We made minor changes to the guidebook to adapt it to an Australian audience (e.g. substituting photos and text relating to snow). 
Tailored-print intervention Participants randomised into this group will be mailed three Social Cognitive Theory-based $[68,69]$ computer-tailored newsletters over a 12 week period (6 weeks apart). Each newsletter will be four A4 pages in length and will provide advice and feedback unique to the individual that relates to key determinants of PA adoption and maintenance among breast cancer survivors (as stipulated by previous research in the field [57,70-77] and Social Cognitive Theory $[68,78])$. The advice participants receive will be tailored using information derived from individual assessments at baseline, and 'update cards' (assessing PA and goal setting behaviour over the last month) sent to participants (in this group only) at 4 weeks and 8 weeks postbaseline. In each case, participants will be mailed the tailored-newsletters within two weeks after the completed assessment is received (see Figure 1).

Newsletter 1 (N1) will include information on the Australian PA guidelines for cancer survivors (non-tailored), tailored feedback on PA behaviour (aerobic, resistance and sitting time) relative to the guidelines, information about the beneficial outcomes of PA, safety advice and an action planning activity. An activity planner and exercise illustrations (stretches and resistance training exercises) will also be included.

Newsletter 2 (N2) will include expert advice from a behaviour change expert (non-tailored), feedback on PA performance (aerobic, resistance and sitting time) relative to N1, a testimonial illustrating success, advice on eliciting social support and an action planning activity.

Newsletter 3 (N3) will include expert advice from an exercise physiologist (non-tailored), tailored feedback on PA performance (aerobic, resistance and sitting time) relative to $\mathrm{N} 2$ and N1, advice on restructuring the physical environment, information about available support services and an action planning activity. See Additional file 1 (Table S1) for a brief overview of how Social Cognitive Theory was operationalised to form these intervention strategies and what variables were used to tailor information.

The tailored-print intervention was developed specifically for this study by following the eight-step procedure outlined by Kreuter et al. [79]. More information is available upon request to the corresponding author and will be available in a separate manuscript.

Standard recommendation control group Participants randomised into this condition will receive the "An active way to better health" brochure published by the Australian government, detailing the national physical activity guidelines for adults [80]. The guidelines stipulate that Australian adults should: (1) think of movement as an opportunity; (2) be active every day in as many ways as you can; (3) do 30 minutes of moderate intensity physical activity on most, preferably all days; and (4) if manageable, do vigorous activity for extra health benefit. A copy of the brochure can be downloaded free of charge from www. healthyactive.gov.au.

\section{Statistical analysis}

Analyses will be conducted according to the intention to treat principal, as outlined by White et al. [81]. Namely, the primary analysis will be conducted using all observed data (i.e., a completers analysis) and sensitivity analyses (accounting for all randomised participants) will be conducted to explore the impact of missing data [81]. Differences between treatment groups in the primary outcome measures (i.e. the two PA scores) 4 months after randomisation will be tested using Analysis of Covariance (ANCOVA). The outcome in the model will be the subjects physical activity score at 4 months and the predictors will be treatment group and baseline value of the physical activity score. In the analysis of each of the two PA measures, if the p-value for the treatment group is less than 0.025 (adjusted to account for the two primary PA analyses) then post hoc tests of the 3 pair wise comparisons will be undertaken to determine which treatment groups are different. Socio-cognitive and QOL measures will also be analysed using ANCOVA models. The study's primary analysis will be the comparison of PA behaviour between the three groups, from baseline to the 4 month time-point. Secondary analyses will examine the PA behaviour change between the study groups across the other study timepoint (i.e., 10 months). A mediation analysis on the employed social-cognitive variables will also be conducted to explore the causal mechanism of any intervention effects. Planned subgroup analyses include age, PA status at baseline, time since treatment, BMI, built environment, and co-morbidity status.

\section{Discussion}

This study will test the relative efficacy of two theorybased PA behaviour change interventions. In doing so, this study will address a seminal research question in distancebased patient-centred care - is tailoring or targeting health education messages a more efficacious approach to health behaviour change in the PA domain? Furthermore, this study will be one of the first to promote a pattern of PA that addresses the metabolic consequences of unbroken sedentary behaviours and the advantages of completing both aerobic and resistance-training exercises. The limitations reported in previous research will be addressed by examining adherence after the intervention period and by utilising an objective measure of PA behaviour (i.e., pedometers). Finally, this study will add to the behaviour change literature by addressing the paucity of knowledge surrounding determinants of PA behaviour change among 
cancer survivors and potential mediators of intervention effects.

\section{Additional file}

Additional file 1: Table S1. Operationalisation of SCT constructs for the Move More for Life intervention.

\section{Competing interests}

The authors declare that they have no competing interests.

\section{Authors' contributions}

$E L J$ and CES conceived the study. CES, AG and RCP obtained the funding. All authors provided input into the study design. CES was primarily responsible for intervention design and recruitment, with significant input from ELJ and RCP. CES, EL and RCP were responsible for drafting the manuscript. PM provided statistical guidance and support and drafted the statistical analyses section of the manuscript. All authors critically evaluated the article for content and approved the final version.

\section{Acknowledgements}

This study was funded from the Cancer Institute New South Wales Research Scholar Award (10/RSA/1-27) awarded to CES. Infrastructure support was provided by the University of Newcastle and the Hunter Medical Research Institute. RCP is supported by a Senior Research Fellowship from the National Health Medical Research Council of Australia.

We thank the multi-disciplinary project advisory group (Ass/Prof Kypros Kypri, University of Newcastle; Dr Sandi Hayes, Queensland University of Technology; Ms Suzanne Mullen, BCNA consumer representative; Ms Mary Macheras-Magias, YWCA Encore; Dr Kendra Sundquist; Cancer Council NSW) who provided input in the early design stages.

We would also like to thank Professor Kerry Courneya and Dr Sandi Hayes for their support in intervention development.

Finally, thank you to Breast Cancer Network Australia, Dragon's Abreast, YWCA Encore and Kimberly Parsons for assistance with recruiting participants.

\section{Author details}

${ }^{1}$ School of Medicine and Public Health, Priority Research Centre for Health Behaviour, Priority Research Centre for Physical Activity and Nutrition, University of Newcastle, Callaghan, Australia. Ingham Institute for Applied Medical Research, South Western Sydney Clinical School, University of New South Wales, Sydney, Australia. ${ }^{3}$ Clinical Trials Unit, Hunter Medical Research Institute, School of Medicine and Public Health, University of Newcastle, Callaghan, Australia. ${ }^{4}$ School of Education, Priority Research Centre for Physical Activity and Nutrition, University of Newcastle, Callaghan, Australia.

Received: 22 March 2012 Accepted: 14 April 2012

Published: 8 May 2012

\section{References}

1. Australian Institute of Health and Welfare, National Breast and Ovarian Cancer Centre: Breast cancer in Australia: an overview, 2009. In Book Breast cancer in Australia: an overview, 2009. City: AlHW; 2009.

2. Aziz NM: Cancer survivorship research: challenge and opportunity. J Nutr 2002, 132:3494S-3503S.

3. Schmitz K, Speck R: Risks and benefits of physical activity among breast cancer survivors who have completed treatment. Womens Health 2010, 6:221.

4. Conn VS, Hafdahl AR, Brown SA, Brown LM: Meta-analysis of patient education interventions to increase physical activity among chronically ill adults. Patient Educ Couns 2008, 70:157-172.

5. Cramp F, Daniel J: Exercise for the management of cancer-related fatigue in adults. Cochrane Database Syst Rev 2008, 16:CD006145.

6. Australian Institute of Health and Welfare \& Australasian Association of Cancer Registries: Cancer in Australia: an overview, 2010. Cancer series no 60th edition. Canberra: AlHW; 2010

7. Baade PD, Fritschi L, Eakin E: Non-cancer mortality among people diagnosed with cancer (Australia). Cancer Causes Control 2006, 17:287-297.
8. Feuerstein M: Handbook of cancer survivorship. New York: Springer Science + Business Media; 2007.

9. Schmitz KH, Courneya KS, Matthews C, Demark-Wahnefried W, Galvao DA, Pinto BM, Irwin ML, Wolin KY, Segal RJ, Lucia A, et al: American College of Sports Medicine roundtable on exercise guidelines for cancer survivors. Med Sci Sports Exerc 2010, 42:1409-1426.

10. Hayes S, Spence RR, Galvao DA, Newton RU: Australian Association for Exercise and Sport Science position stand: optimising cancer outcomes through exercise. J Sci Med Sport2009, 12:428-434.

11. Aziz NM, Rowland JH: Trends and advances in cancer survivorship research: challenge and opportunity. Semin Radiat Oncol 2003, 13:248-266.

12. Chlebowski R: Lifestyle change including dietary fat reduction and breast cancer outcome. J Nutr 2007, 137:233S-235S.

13. Holick CN, Newcomb PA, Trentham-Dietz A, Titus-Ernstoff L, Bersch AJ, Stampfer MJ, Baron JA, Egan KM, Willett WC: Physical activity and survival after diagnosis of invasive breast cancer. Cancer Epidemiol Biomarkers Prev 2008, 17:379-386.

14. Holmes M, Chen W, Feskanich D, Kroenke C, Colditz G: Physical activity and survival after breast cancer diagnosis. JAMA 2005, 25:2479-2486.

15. Lynch BM: Sedentary behavior and cancer: a systematic review of the literature and proposed biological mechanisms. Cancer Epidemiol Biomarkers Prev 2010, 19:2691-2709.

16. Courneya KS, Mackey JR, McKenzie DC: Exercise for breast cancer survivors: research evidence and clinical guidelines. Physician and Sportsmedicine 2002, 30:33-42.

17. Lynch B, Dunstan D, Healy G, Winkler E, Eakin E, Owen N: Objectively measured physical activity and sedentary time of breast cancer survivors, and associations with adiposity: findings from NHANES (20032006). Cancer Causes Control 2010, 21:283-288.

18. Eakin EG, Youlden DR, Baade PD, Lawler SP, Reeves MM, Heyworth JS, Fritschi L: Health behaviours of cancer survivors: data from an Australian population-based survey. Cancer Causes Control 2007, 18:881-894.

19. Jones LW, Courneya KS: Exercise counseling and programming preferences of cancer survivors. Cancer Pract 2002, 10:208-215.

20. Young-McCaughan S, Sexton DL: A retrospective investigation of the relationship between aerobic exercise and quality of life in women with breast cancer. Oncol Nurs Forum 1991, 18:751-757.

21. Segar ML, Katch VL, Roth RS, Garcia AW, Portner TI, Glickman SG, Haslanger S, Wilkins EG: The effect of aerobic exercise on self-esteem and depressive and anxiety symptoms among breast cancer survivors. Oncol Nurs Forum 1998, 25:107-113.

22. Demark-Wahnefried W, Peterson B, McBride C, Lipkus I, Clipp EC: Current health behaviours and readiness to pursue life-style changes among men and women diagnosed with early stage prostate and breast carcinomas. Cancer 2000, 88:674-684.

23. Courneya K: Physical activity in cancer survivors: a field in motion. Psychooncology 2009, 18:337-342.

24. Pollard A, Eakin E, Vardy J, Hawkes A: Health behaviour interventions for cancer survivors: An overview of the evidence and contemporary Australian trials. Cancer Forum 2009, 33

25. Holtzman J, Schmitz K, Babes G, Kane RL, Duval S, Wilt TJ, MacDonald RM, Rutks I: Effectiveness of behavioral interventions to modify physical activity behaviors in general populations and cancer patients and survivors. In Evididence Report/ Technology Assessment (Prepared by the Minnesota Evidence-based Practice Center, under Contract No. 290-02-0009.) AHRQ Publication No. 04-E027-2. Rockville: Agency for Healthcare Research and Quality; 2004.

26. Pinto B, Floyd A: Theories underlying health promotion interventions among cancer survivors. Semin Oncol Nurs 2008, 24:153-163.

27. Kim C-J, Kang D-H, Park J-W: A meta-analysis of aerobic exercise interventions for women with breast cancer. West J Nurs Res 2009, 31:437-461.

28. Conn VS, Hafdahl AR, Porock DC, McDaniel R, Nielsen PJ: A meta-analysis of exercise interventions among people treated for cancer.[erratum appears in Support Care Cancer.2007 Dec;15(12):1441-2]. Support Care Cancer 2007, 2006(14):699-712. 
29. McNeely ML, Campbell KL, Rowe BH, Klassen TP, Mackey JR, Courneya KS: Effects of exercise on breast cancer patients and survivors: a systematic review and meta-analysis. CMAJ: Canadian Medical Association Journal 2006, 175:34-41.

30. Glanz K, Rimer BK, Lewis FM (Eds): Health behaviour and health education: Theory, research and practice. 3 rd editionth edition. San Franscisco: JosseyBass; 2002.

31. Nutbeam D: Improving the fit between research and practice in health promotion: overcoming structural barriers. Can J Public Health 1996, 87:S18-23.

32. Napolitano MA, Marcus BH: Targeting and tailoring physical activity information using print and information technologies. Exerc Sport Sci Rev 2002, 30:122-128.

33. Short C, James E, Plotnikoff R, Girgis A: Efficacy of tailored-print interventions to promote physical activity: a systematic review of randomised trials. Int J Behav Nutr Phys Act

2011, 8:113.

34. Petty $R$, Cacioppo J: The effects of involvement on the responses to argument quantity and quality: central and peripheral routes to persuasion. J Pers Soc Psychol 1984, 46:69-81.

35. Glasgow RE, McKay HG, Piette JD, Reynolds KD: The RE-AIM framework for evaluating interventions: what can it tell us about approaches to chronic illness management? Patient Educ Couns2001, 44:119-127.

36. Moher D, Hopewell S, Schulz K, Montori V, Gotzsche P, Devereaux P, Elbourne D, Egger M, Altman D: CONSORT 2010 Explanation and Elaboration: updated guidelines for reporting parallel group randomised trials. BMJ 2010, 340:c869.

37. Harrington NG, Noar SM: Reporting standards for studies of tailored interventions. Health Educ Res 2012, 27:331-342.

38. American College of Sports Medicine: ACSM's Guidelines for Exercise Testing and Prescription. 6th edition. New York: Lippincott, Williams, \& Wilkins; 2000

39. Altman DG, Bland JM: How to randomise. Br Med J 1999, 319:703-704

40. Schulz KF: Subverting randomization in controlled trials. JAMA 1995, 274:1456-1458

41. Cohen J: Statistical power analysis for the behavioral sciences. 2nd edition. Hillsdale: Lawrence Earlbaum Associates; 1988

42. Bravata D, Smith-Spangler C, Sundaram V, Gienger A, Lin N, Lewis R, Stave C, Olkin I, Sirard J: Using pedometers to increase physical activity and improve health: a systematic review. JAMA 2007, 21:2296-2304.

43. Tudor-Locke C, Washington T, Hart T: Expected values for steps/day in special populations. Prev Med 2009, 49:3-11.

44. Liebreich T, Plotnikoff R, Courneya K, Boule N: Diabetes NetPLAY: A physical activity website and linked email counselling randomized intervention for individuals with type 2 diabetes. Int J Behav Nutr Phys Act 2009, 6:18.

45. Godin G, Shepard R: Godin Leisure-Time Exercise Questionnaire. Medicine \& Science in Sports \& Exercise 1997, 29:36-38.

46. Plotnikoff RC, Taylor LM, Wilson PM, Courneya KS, Sigal RJ, Birkett N, Raine KIM, Svenson LW: Factors Associated with Physical Activity in Canadian Adults with Diabetes. Medicine \& Science in Sports \& Exercise 2006, 38:1526-1534.

47. Jacobs D, Ainsworth B, Hartman T, Leon A: A simultaneous evaluation of 10 commonly used physical activity questionnaires. Med Sci Sports Exerc 1993, 25:81-91.

48. Tudor-Locke CE, Myers AM: Methodological considerations for researchers and practitioners using pedometers to measure physical (ambulatory) activity. Res Q Exerc Sport 2001, 72:1-12.

49. Tudor-Locke C, Burkett L, Reis JP, Ainsworth BE, Macera CA, Wilson DK: How many days of pedometer monitoring predict weekly physical activity in adults? Prev Med 2005, 40:293-298.

50. Tudor-Locke C, Bassett DR, Shipe MF, McClain JJ: Pedometry methods for assessing free-living adults. J Phys Act Health 2011, 8:445-453.

51. Miller R, Brown WJ, Tudor-Locke C: But what about swimming and cycling? How to count non-ambulatory activity when using pedometers to assess physical activity. J Phys Act Health 2006, 3:257-266

52. Marshall AL, Miller YD, Burton NW, Brown WJ: Measuring Total and Domain-Specific Sitting: A Study of Reliability and Validity. Medicine \& Science in Sports \& Exercise 2009, 42:1094-1102

53. Cella DF, Tulsky DS, Gray G, Sarafian B, Linn E, Bonomi A, Silberman M, Yellen SB, Winicour P, Brannon J, et al: The Functional Assessment of
Cancer Therapy scale: development and validation of the general measure. J Clin Oncol 1993, 11:570-579.

54. Yellen S B, Cella D, F, Webster K, Blendowski C, Kaplan E: Measuring fatigue and other anemia-related symptoms with the Functional Assessment of Cancer Therapy (FACT) measurement system. J Pain Symptom Manage 1997, 13:63-74

55. Rogers LQ, Matevey C, Hopkins-Price P, Shah P, Dunnington G, Courneya KS: Exploring social cognitive theory constructs for promoting exercise among breast cancer patients. Cancer Nurs 2004, 27:462-473.

56. Plotnikoff RC, Blanchard CM, hotz SB, Rhodes R: Validation of the decisional balance scales in the exercise domain from the transtheoretical model: a longitudinal test. Measurement in Physical Education \& Exercise Science 2001, 5:191.

57. Rogers L, Shah P, Dunnington G, Greive A, Shanmugham A, Dawson B, Courneya K: Social cognitive theory and physical activity during breast cancer treatment. Oncol Nurs Forum 2005, 32:807-815.

58. Lewis C, Raczynski J, Heath G, Levinson R, Hilyer JJ, Cutter G: Promoting physical activity in low-income African-American communities: the PARR project. Ethn Dis 1993, 3:106-118.

59. Rogers L, Courneya K, Verhulst S, Markwell S, Lanzotti V, Shah P: Exercise barrier and task self-efficacy in breast cancer patients during treatment. Support Care Cancer 2006, 14:84-90.

60. Plotnikoff RC, Lippke S, Courneya KS, Birkett N, Sigal RJ: Physical activity and social cognitive theory: a test in a population sample of adults with type 1 or type 2 diabetes. Applied Psychology 2008, 57:628-643.

61. Sallis JF, Grossman RM, Pinski RB, Patterson TL, Nader PR: The development of scales to measure social support for diet and exercise behaviors. Prev Med 1987, 16:825-836.

62. Spittaels H, Foster C, Oppert J-M, Rutter H, Oja P, Sjostrom M, De Bourdeaudhuij I: Assessment of environmental correlates of physical activity: development of a European questionnaire. Int J Behav Nutr Phys Act 2009, 6:39.

63. Umstattd MR, Motl R, Wilcox S, Saunders R, Watford M: Measuring physical activity self-regulation strategies in older adults. J Phys Act Health 2009, 6: S105-S112.

64. Rise J, Thompson M, Verplanken B: Measuring implementation intentions in the context of the theory of planned behavior. Scand J Psychol 2003, 44:87-95.

65. Rhodes RE, Blanchard CM, Matheson DH, Coble J: Disentangling motivation, intention, and planning in the physical activity domain. Psychology of Sport and Exercise 2006, 7:15-27.

66. Vallance JKH, Courneya KS, Plotnikoff RC, Yasui Y, Mackey JR: Randomized controlled trial of the effects of print materials and step pedometers on physical activity and quality of life in breast cancer survivors. J Clin Oncol 2007, 25:2352-2359.

67. Vallance JK, Courneya KS, Taylor LM, Plotnikoff RC, Mackey JR: Development and evaluation of a theory-based physical activity guidebook for breast cancer survivors. Health Educ Behav 2008, 35:174-189.

68. Bandura A: Health promotion by social cognitive means. Health Educ Behav 2004, 31:143-164.

69. Bandura A: Self-efficacy: toward a unifying theory of behavioral change. Psychol Rev 1977, 84:191-215

70. Krebs P, Prochaska JO, Rossi JS: Defining what works in tailoring: a meta-analysis of computer-tailored interventions for health behavior change. Prev Med 2010, 51:214-221.

71. Noar S, Benac CN, Harris MS: Does tailoring matter? Meta-analytic review of tailored print health behavior change interventions. Psychol Bull 2007 133:673-693.

72. Michie S, Abraham C, Whittington C, McAteer J, Gupta S: Effective techniques in healthy eating and physical activity interventions: a meta-regression. Health Psychol2009, 28:690-701.

73. Rogers A, Courneya KS, Verhulst S, Markwell SJ, McAuley E: Factors associated with exercise counselling and program preferences among breast cancer survivors. Journal of Physical Activity and Health 2008, 5:688-705.

74. Rogers LQ, Courneya KS, Shah P, Dunnington G, Hopkins-Price P: Exercise stage of change, barriers, expectations, values and preferences among breast cancer patients during treatment: a pilot study. Eur J Cancer Care 2007, 16:55-66. 
75. Park CL, Gaffey AE: Relationships between psychosocial factors and health behavior change in cancer survivors: an integrative review. Ann Behav Med 2007, 34:115-134.

76. Pinto BM, Ciccolo JT: Physical activity motivation and cancer survivorship. Recent Results Cancer Res 2011, 186:367-387.

77. Harrison S, Hayes SC, Newman B: Level of physical activity and characteristics associated with change following breast cancer diagnosis and treatment. Psychooncology 2009, 18:387-394.

78. Bandura A: Social Foundations of Thought and Action: A Social Cognitive Theory. New York: Prentice Hall; 1986.

79. Kreuter M, Farrell D, Olevitch L, Brennan L: Tailoring health messages: Customizing communication with computer technology Mahwah. New Jersey: Lawrence Erlbaum Associates; 2000.

80. Department of Health and Aged Care: An active way to better health: National physical activity guidelines for adults. Canberra: Australian Government; 1999

81. White IR, Horton NJ, Carpenter J, Pocock SJ: Strategy for intention to treat analysis in randomised trials with missing outcome data. Br Med J 2011, $7: 342$.

doi:10.1186/1471-2407-12-172

Cite this article as: Short et al: Move more for life: the protocol for a randomised efficacy trial of a tailored-print physical activity intervention for post-treatment breast cancer survivors. BMC Cancer 2012 12:172.

\section{Submit your next manuscript to BioMed Central and take full advantage of:}

- Convenient online submission

- Thorough peer review

- No space constraints or color figure charges

- Immediate publication on acceptance

- Inclusion in PubMed, CAS, Scopus and Google Scholar

- Research which is freely available for redistribution 